\title{
chaJOPEN
}

\section{How the experience of medical assistance in dying changed during the COVID-19 pandemic in Canada: a qualitative study of providers}

\author{
Ellen Wiebe MD, Michaela Kelly MSc, Thomas McMorrow DCL, Sabrina Tremblay-Huet LLB, \\ Brian Sum MD, Mirna Hennawy BSc
}

Abstract

Background: In March 2020, all levels of government introduced various strategies to reduce the impact of the COVID-19 pandemic. The purpose of this study was to document how the experience of providing medical assistance in dying (MAiD) changed during the COVID-19 pandemic.

Methods: We conducted a qualitative study using semistructured interviews with key informants in Canada who provided or coordinated MAiD before and during the COVID-19 pandemic. We interviewed participants from April to June 2020 by telephone or email. We collected and analyzed data in an iterative manner and reached theme saturation. Our team reached consensus on the major themes and subthemes.

Results: We interviewed 1 MAiD coordinator and 15 providers, including 14 physicians and 1 nurse practitioner. We identified 4 main themes. The most important theme was the perception that the pandemic increased the suffering of patients receiving MAiD by isolating them from loved ones and reducing available services. Providers were distressed by the difficulty of establishing rapport and closeness at the end of life, given the requirements for physical distancing and personal protective equipment. They were concerned about the spread of SARS-CoV-2, and found it difficult to enforce rules about distancing and the number of people present. Logistics and access to MAiD became more difficult because of the new restrictions, but there were many adaptations to solve these problems.

Interpretation: Providers and coordinators had many challenges in providing MAiD during the COVID-19 pandemic, including their perception that the suffering of their patients increased. Some changes in how MAiD is provided that have occurred during the pandemic, including more telemedicine assessments and virtual witnessing, are likely to remain after the pandemic and may improve service.

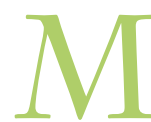
edical assistance in dying (MAiD) has been available in Canada since 2016, when the law was changed based on a constitutional challenge. ${ }^{1,2}$ The federal government reported that over 13000 Canadians chose this option between 2016 and the end of $2019 .^{3}$ Medical assistance in dying includes medication injected by a provider to end life; it also includes self-ingested lethal medication in all provinces except Quebec. ${ }^{4}$ The patient must meet all legal eligibility criteria; during the study period, that included being over 18 years of age and having a grievous and irremediable medical condition, from which a natural death is reasonably foreseeable. ${ }^{1}$

The first case of COVID-19 in Canada was diagnosed in a care home on Jan. 20, 2020.5 Over the months that followed, the provinces ordered various strategies to reduce deaths from the virus and strain on the health system. ${ }^{6}$ These varied by province, but included travel restrictions, reduction or banning of visitors from health facilities, banning transfers from one health institution to another, virus testing, quarantining and self-isolation. By Mar. 31, 2021, there had been over 982116 cases of COVID-19 and over 22959 deaths in Canada. ${ }^{7}$ The pandemic changed many aspects of providing medical care, including an increased use of virtual care with video or audio technology, reduced elective procedures and redeployment of staff from one area or institution to another. ${ }^{6,8,9}$

In this study, we explored the effects of the pandemic on providing MAiD in Canada. Our research team had been exploring various aspects of the experience of MAiD in Canada, and so we were able to pivot quickly to start asking key informants how the provision of MAiD had changed during the pandemic. Our primary objectives were to understand the

Competing interests: None declared.

Correspondence to: Ellen Wiebe, ellenwiebe@gmail.com

CMAJ Open 2021. DOI:10.9778/cmajo.20200163 
impact of the pandemic on MAiD care and to identify the strategies used to reduce these impacts.

\section{Methods}

\section{Setting and recruitment}

This was a qualitative study using semistructured interviews of MAiD providers and coordinators across Canada. We used convenience and purposive recruitment strategies, including snowball sampling (i.e., we asked participants if they would pass our request on to other key informants), to collect information quickly from key informants. The participants were recruited from electronic mailing lists of providers and from a list of previous research participants who had consented to further interviews about their experience with MAiD. ${ }^{10}$ The mailing lists include about 180 providers, each of whom was screened by the sponsoring organizations (Canadian Association of MAiD Assessors and Providers and Aide Médicale à Mourir). With these recruitment strategies, we intended to access key informants who would be best situated to articulate any changes they experienced in providing MAiD care during the COVID-19 pandemic.

We emailed potential and previous research participants an invitation to participate in an interview. The letter shared the purpose of the study and the backgrounds of the research team. A follow-up email was sent 2 weeks after the initial invitation. People were eligible to participate if they were a MAiD assessor, provider or care coordinator, and if they had experience with at least 1 patient receiving MAiD before the COVID-19 pandemic and at least 1 patient during the pandemic.

\section{Data collection}

The semistructured interview guide was designed by the research team based on information from the Canadian Association of MAiD Assessors and Providers and information in the media about changing health care regulations during the pandemic (Appendix 1, available at www.cmajopen.ca/ content/9/2/E400/suppl/DC1). We developed the guide in English, and 1 team member (S.T.-H.) translated it into French. Three team members conducted the interviews in either English (B.S. and M.H.) or French (S.T.-H.) using video, audio or email between Apr. 16 and June 5, 2020. The interviewers had previous experience with qualitative research and were familiar with MAiD practices in Canada. Using semistructured interviews allowed for discussion of the predetermined topics in the guide and for additional questions to emerge from the conversation. ${ }^{11} \mathrm{We}$ audio-recorded the video and audio interviews and transcribed them verbatim. One team member (S.T.-H.) translated the French transcripts to English so that all investigators could participate in analysis.

We collected and analyzed data in an iterative manner, with data collection and analysis occurring simultaneously. ${ }^{11}$ Analysis of the initial interviews began as soon as they were complete, which allowed us to change the interview guide to explore any new topics that our participants introduced. ${ }^{11}$ Once all interviews were complete, we emailed each participant a copy of their transcripts for approval. We also asked if they had any new or different experiences since the time of their interview that they wanted to share with us.

All participants provided informed consent before the interview. Deidentified transcripts were stored on a secure site shared only with investigators, and audio files were deleted once transcription was complete.

\section{Data analysis}

We used abductive reasoning to identify main themes on the basis of their recurrence across all of the interviews, as well as their prominence in each interview. ${ }^{12}$ Adopting this approach meant tracing overlap among the situations our informants described and the concerns they expressed, while attending to specific details in their accounts. The purpose of deploying abductive reasoning is to draw reasonable inferences based on the available data of how MAiD providers have experienced the COVID-19 pandemic. To support our abductive reasoning, we developed a codebook and used it to tag segments of the interviews, sort similar segments into categories and organize these categories into themes. One author (M.H.) developed the codebook, and we edited it as a team throughout the concurrent data collection and analysis process until we reached consensus on the major themes and subthemes. We reached theme saturation (i.e., no more new themes were emerging) before we completed data collection but continued to interview participants to get a more representative sample. Three authors (M.H., B.S. and E.W.) coded the transcripts. Participants were not a part of the data analysis process.

\section{Ethics approval}

This study was approved by the research ethics boards of the University of British Columbia (H15-03198-A018).

\section{Results}

Sixteen people responded to our letter of invitation and all of these respondents participated in interviews. One participant preferred to complete the interview by email, 13 by phone and 2 by video. All participants preferred to respond to the follow-up question by email. Interviews ranged from 10 to 45 minutes (mean 32 minutes). We reached theme saturation after 8 interviews, but we continued interviewing to gain the experience of more participants.

Our participants included $15 \mathrm{MAiD}$ providers and $1 \mathrm{MAiD}$ care coordinator (Table 1). Fourteen providers were physicians and 1 was a nurse practitioner. Their ages ranged from 28 to 82 (mean age 54.5 ) years, and they lived in 6 provinces (Alberta, British Columbia, Manitoba, Ontario, Quebec and Saskatchewan). Three were from Quebec and 13 were from the rest of Canada (ROC). Of the 16 participants, 10 worked in an urban setting, 4 worked in a rural setting and 2 worked in a mix of both urban and rural settings. The specialties of the providers included primary care, geriatrics, emergency medicine, palliative care and anesthesiology. ${ }^{1,10}$

The 15 MAiD providers had completed between 1 and 460 (mean 133.1) MAiD assessments before the COVID-19 pandemic, and between 2 and 20 (mean 8.2) MAiD assessments 


\begin{tabular}{|c|c|}
\hline Characteristic & $\begin{array}{l}\text { No. }(\%) \text { of participants* } \\
n=16\end{array}$ \\
\hline \multicolumn{2}{|l|}{ Gender } \\
\hline Female & $13(81)$ \\
\hline Male & $3(19)$ \\
\hline \multicolumn{2}{|l|}{ Age, yr } \\
\hline $25-34$ & $1(6)$ \\
\hline $35-44$ & $3(19)$ \\
\hline $45-54$ & $4(25)$ \\
\hline $55-64$ & $3(19)$ \\
\hline $65-74$ & $3(19)$ \\
\hline $75-84$ & $2(12)$ \\
\hline \multicolumn{2}{|l|}{ Profession } \\
\hline Physician & $14(88)$ \\
\hline Nurse practitioner & $1(6)$ \\
\hline MAiD care coordinator & $1(6)$ \\
\hline \multicolumn{2}{|l|}{ Province } \\
\hline British Columbia & $5(31)$ \\
\hline Alberta & $1(6)$ \\
\hline Manitoba & $1(6)$ \\
\hline Saskatchewan & $1(6)$ \\
\hline Quebec & $3(19)$ \\
\hline Ontario & $5(31)$ \\
\hline \multicolumn{2}{|l|}{ Practice setting } \\
\hline Urban & $10(62)$ \\
\hline Rural & $4(25)$ \\
\hline Mixed & $2(12)$ \\
\hline \multicolumn{2}{|l|}{ Specialty } \\
\hline Primary care & $11(69)$ \\
\hline Palliative care & $1(6)$ \\
\hline Anesthesiology & $1(6)$ \\
\hline Geriatrics & $1(6)$ \\
\hline Emergency medicine & $1(6)$ \\
\hline NA & $1(6)$ \\
\hline \multicolumn{2}{|c|}{$\begin{array}{l}\text { No. of MAiD assessments before } \\
\text { COVID-19 (providers only) }\end{array}$} \\
\hline Mean (range) & $133.1(1-460)$ \\
\hline Median & 100 \\
\hline \multicolumn{2}{|c|}{$\begin{array}{l}\text { No. of MAiD assessments during } \\
\text { COVID-19 (providers only) }\end{array}$} \\
\hline Mean (range) & $8.2(2-20)$ \\
\hline Median & 7 \\
\hline
\end{tabular}

during the pandemic. The MAiD care coordinator had coordinated about 500 cases before the COVID-19 pandemic and 55 cases during the pandemic.

\section{Themes}

Four main themes emerged during analysis of the data: increased patient suffering, increased challenges in establishing rapport, anxiety about spreading SARS-CoV-2, and individual and institutional adaptations.

The most important theme, the one for which our participants used the most emotionally laden language, was the increased suffering they perceived in their patients requesting MAiD (Box 1). This theme included the following subthemes: patients being unable to spend time with loved ones because of institutional visiting policies and travel restrictions, proce-

\section{Box 1: Increased suffering for patients}

Being unable to spend time with loved ones

- ' 'It's so sad. They are so sad that they just couldn't be with a family member. And you have to work really hard to get anyone in, and the hospital doesn't want to let them in until it's the very end, and that's terrible." (Participant 14, age 64 years, physician)

- "When l've done the assessment and I'm talking to the patients' families, they talk about how horrible that they can't visit, or if they're at home, that they worry their loved one is going to have to go to hospital, and then they can't visit or find out what's going on." (Participant 11, age 36 years, physician)

- "So a lot of people, their reasons for living was spending a lot of time with their family and grandkids and whoever, and that was no longer possible for them." (Participant 8 , age 48 years, physician)

Having procedures cancelled or rescheduled

- "So we were going to do it about 6 or 7 days after because we didn't think that he was going to make it through, but then [city name] decided to cancel MAiD at that time, and our hospital followed, so they wouldn't let me proceed with it." (Participant 10 , age 76 years, physician)

- "I had one who basically needed to be in the hospital, and when the visitor restrictions came into place, they were advised that it was for the safety of the patient and also for her family, because there was COVID in the hospital, she didn't want to put her family at risk and she didn't want to be alone, so she decided that it was time to die." (Participant 8 , age 48 years, physician)

Being unable to offer other end-of-life services

- "So there's 3 kind of palliative care places in [City name] and 2 of them are closed now, because of outbreaks of COVID, so there really is no place for people to go." (Participant 12 , age 74 years, physician)

- "So she was having severe symptoms that were being only partially controlled and normally should have gone straight to the palliative care ward, but no transfers were allowed to either the hospice or the palliative care ward. [...]. I mean she was so uncomfortable when I saw her at home. It was awful. But she decided that she had to go home, because that was the only way she could see her son." (Participant 2, age 67 years, physician)

Note: $\mathrm{MAiD}=$ medical assistance in dying. 
dures being cancelled or rescheduled because of changes in institutional policies and being unable to offer other end-oflife services, like residential hospice care.

The second theme was about the increased challenges for providers in establishing rapport when using telemedicine assessments and when using personal protective equipment for in-person care. Some providers said they felt a decreased closeness because they were unable to touch or hug at the time of provision of MAiD (Box 2).

Another theme was provider anxiety about the spread of SARS-CoV-2 and about observance of the relevant public health rules and institutional policies. Participants reported that, at times, there were too many people present and too little distance when MAiD was being provided. Our participants varied in their comfort level with this rule breaking, but all participants had experience with it. There was a tension between providing the best patient-centred care and respecting public health imperatives (Box 3).

The fourth theme was that, although logistics and access to MAiD became more difficult with the new COVID-19 restrictions, people and institutions adapted to mitigate the problems around providing MAiD. Providers talked about

Box 2: Increased challenges in establishing rapport when conducting telemedicine assessments, wearing personal protective equipment and being unable to have physical contact

- "The last one, the assessment was done virtually, yeah, and you just feel a little bit removed from it. I felt badly about it. You know it's harder, I find it harder to connect with people, you know, on the phone, obviously, or even in person when you're wearing all the gear." (Participant 12, age 74 years, physician)

- "It was weird that I had to be all suited up to see her at a time when I wanted to connect, when I want my face to be seen to really connect with somebody at the last minutes of their life." (Participant 2, age 67 years, physician)

- "Well, people have a harder time hearing us because a lot of older people, you lose high frequency hearing as you get older, and the way older people compensate for that is they watch the mouth and they actually been lip-reading the whole time. And so, lip-reading lets them just figure out what somebody's saying. And so with a mask on, they can't actually do that very well and so, it does impede it. And you can't get quite as much of that interpersonal connection either with the mask." (Participant 14, age 64 years, physician)

- "So the first patient that I saw was early on in COVID and I had to maintain the 2-m distance and I just felt that I couldn't get close, I didn't feel close, emotionally to her, because I like to hold their hand when I greet them, get a little closer, look at them, smile, and I like to make that connection at the beginning and I couldn't do that." (Participant 3, age 65 years, physician)

- "Especially the fact of not being able to embrace, to shake hands ... the frustration and the embarrassment are as much on the patient's side as on mine. My MAiD cases are always intense and emotional." (Participant 13, age 74 years, physician)

Note: $\mathrm{MAiD}=$ medical assistance in dying. how some institutions made exceptions to their rules for endof-life care and how helpful virtual care could be for mitigating the difficult logistics of providing MAiD, both during the pandemic and moving forward (Box 4).

\section{Interpretation}

Our participants described how COVID-19 had changed the way they provided MAiD. They had experience before and

\section{Box 3: Anxiety about spreading SARS-CoV-2}

- 'There's supposed to be 2 people there, her husband and her son, but then when I went in there, there were like 5 people there, which I couldn't, didn't feel like I could do anything about because, you know, it was another son and her mother, like you can't really say that they can't. I couldn't, didn't feel like I could say they couldn't be there." (Participant 12, age 74 years, physician)

- "It was difficult to go back and do the provision and not be able to be physically close, but I actually broke the rules, you know, I did come closer to the wife, I was able to hold her hand and she appreciated that. I did sit beside the patient to get his consent, there was no 6 feet there. So I found that I was breaking the rules." (Participant 3 , age 65 years, physician)

- "Am I putting myself at risk of COVID, entering his very small apartment and he had a way of speaking that really projected spit, a lot, and so I had to stay further from him, which felt very impersonal, and I had to wear a mask." (Participant 5, age 57 years, physician)

Box 4: Individual and institutional adaptations to mitigate challenges of providing MAiD during the COVID-19 pandemic

- "They allowed several family members to come. They moved the patient to a private room where they had the room that had its own entry point so the family wouldn't have to walk through the entire unit, they would just be able to come to the outside and come through this one door." (Participant 1, age 45 years, coordinator)

- 'So, we've always done some video assessments, so it's not completely new, but we used to only do those for people that lived far away, and now we're doing basically as many as possible via video." (Participant 11, age 36 years, physician)

- "The patient wasn't late stage enough to qualify for visitors as an end of life case, but as soon as they applied for MAiD and set a date, then they were allowed visitors suddenly when they weren't before, and the family had a lot of distress over not being able to visit beforehand, so they were grateful to be allowed in for the time they had left." (Participant 8, 48 years, physician)

- 'I think technology is great and I think that we've had to adapt so quickly. There's so many like bureaucratic bull ${ }^{\star * \star *}$ that we've been able to get through so much quicker than we ever would have been able to before. The stuff we're able to do right now is so phenomenal and I hope we can keep some of it when COVID eases or leaves us at some point. I hope we can keep some of the virtual witnessing and virtual work that we're able to do." (Participant 7 , age 39 years, nurse practitioner)

Note: $\mathrm{MAiD}=$ medical assistance in dying. 
during the COVID-19 pandemic and were able to make comparisons. The most important theme was the increased suffering they saw in their patients, which we expect to be true of all patients at the end of life during the COVID-19 pandemic. This was anticipated and has been seen in previous pandemics. ${ }^{13,14}$ The many rules in place to prevent the spread of SARS-CoV-2 have meant increased loneliness and isolation of older adults in hospitals, in care homes and at home. ${ }^{6,7}$ Their family members and friends cannot visit, volunteers are not allowed and all the social programming in care homes and communities has stopped. The rules preventing transfers between facilities and between units in a given facility interfered with some patients getting palliative care or MAiD.

The providers in our study were distressed by the difficulty they experienced in establishing rapport and in feeling close to their patients at the end of life, because they were required to distance and wear personal protective equipment. They were used to touching and even hugging their patients and family members. Many people at the end of life have agerelated hearing loss and other conditions that interfere with communication, so the mandatory use of masks made important communication even more challenging. Communication has been shown to be important in satisfaction with all types of medical care, but especially at the end of life. ${ }^{15}$

The COVID-19 pandemic has accentuated existing problems in MAiD provision. Before the pandemic, the challenges in getting witnesses for the request form, the lack of providers in certain areas and the faith-based facilities that refused to allow MAiD all presented difficulties to patients requesting MAiD. During the COVID-19 pandemic, all in-person volunteer witnessing stopped, and it took some time to establish virtual witnessing using video platforms. ${ }^{16}$ Some providers were redeployed to COVID-19 work and were unavailable for MAiD services, and others were quarantined because of symptoms consistent with SARS-CoV-2 infection or contact with a person with COVID-19. Some secular hospitals stopped MAiD programs during the pandemic and faith-based facilities did not change their policies to start allowing MAiD. ${ }^{17}$ Transfers between facilities were no longer allowed, interfering with end-of-life choices for some patients. ${ }^{6}$

There were many logistical challenges to providing MAiD during the COVID-19 pandemic, including sourcing personal protective equipment, using more telemedicine in situations better suited to in-person visits and trying to keep safe in situations where family members wanted to be close. Providers and their institutions also developed new systems and protocols to adapt to the challenges posed by the pandemic. Some of these adaptations were very helpful and will remain in place after the pandemic is over. For example, although telemedicine assessments are not ideal for every patient, more virtual witnessing and assessing could decrease delays and decrease travelling for both patients and providers. ${ }^{15}$ This is particularly valuable for patients at the end of life, for whom getting to appointments outside of their home or community may be burdensome. ${ }^{18}$ When there was a looming shortage of the medication, propofol, used for both patients with COVID-19 and those receiving MAiD, the appropriate authorities changed protocols to prevent wastage. ${ }^{19}$ These protocols are likely to be maintained moving forward. Our findings may be used to improve care for patients requesting MAiD during the COVID-19 pandemic and to inform regulation-making during the pandemic and future health crises.

\section{Limitations}

Limitations of this study include the small number of providers and coordinators interviewed. Coordinators have different roles in different jurisdictions but generally provide information to patients and providers and help organize assessments. We do not know why more coordinators did not respond. There are many more physicians who provide MAiD than nurse practitioners, so it was not surprising to have only 1 nurse practitioner participate. Many health jurisdictions, including all of Quebec, do not have nurse practitioners who provide MAiD. Additionally, the voices of patients and their support people were not included in this study; it would be valuable for future research to explore the perspectives of these groups. We did not back-translate the interviews, but because our transcripts were translated by 1 of the investigators (S.T.-H.) doing the analysis, and a second investigator (T.M.) was also fluently bilingual, we were able to get full explanations of both language usage and cultural subtleties in the language.

There are no data available on the number of MAiD providers and coordinators in Canada, so it is difficult to estimate whether or not our sample was representative, though our sample includes different genders, age groups, practice types and practice locations. More importantly, most participants were experienced, active providers, having cumulatively completed over 2000 assessments. We started the study Apr. 16, 2020, only 35 days after the first COVID-19 restrictions were in place, but we did ask each participant to review their interview transcript at the end of May and add anything they thought was important. By the end of the data collection on June 5, 2020, some of the restrictions had been eased. This means that we did not document the full range of COVID-19 restrictions, but we were able to capture the experience of the most restrictive period during the first wave of the COVID-19 pandemic.

\section{Conclusion}

The MAiD providers and coordinators in this study perceived that the restrictions put in place to mitigate the COVID-19 pandemic increased the suffering of their patients seeking MAiD. People are isolated from loved ones, there are fewer services available and the requirements for personal protective equipment interfere with establishing rapport and closeness with health care workers. Access to MAiD has been more limited during the COVID-19 pandemic and the logistics of obtaining assessments, sourcing medications and finding appropriate locations have become more cumbersome. Some changes that have occurred, including more telemedicine assessments and virtual witnessing, are likely to remain after the pandemic and have the potential to improve service. 


\section{References}

1. Legislative background: Medical Assistance in Dying (Bill C-14). Ottawa: Department of Justice Canada; 2016. Available: www.justice.gc.ca/eng/rp-pr/ other-autre/ad-am/ad-am.pdf (accessed 2020 May 19).

2. Beschle D. Carter v. Canada (Attorney General): Canadian courts revisit the criminalization of assisted suicide. Wayne Law Rev 2013;59:561-87.

3. Fourth interim report on medical assistance in dying in Canada. Ottawa: Health Canada; 2019. Available: www.canada.ca/en/health-canada/services/ publications/health-system-services/medical-assistance-dying-interim-report -april-2019.html (accessed 2020 Apr. 17).

4. Act Respecting End-of-life Care, RLRQ, chapter S-32.0001.

5. Coronavirus disease 2019 (COVID-19): epidemiological update. Ottawa: Government of Canada; 2020. Available: https://health-infobase.canada.ca/covid-19/ epidemiological-summary-covid-19-cases.html? topic=ex-col-faq (accessed 2020 May 19).

6. COVID-19 (novel coronavirus). Government of British Columbia; 2020. Available: https://www2.gov.bc.ca/gov/content/health/about-bc-s-health-care -system/office-of-the-provincial-health-officer/current-health-topics/covid-19 -novel-coronavirus (accessed 2020 May 19).

7. Coronavirus disease (COVID-19): Outbreak update. Ottawa: Government of Canada. Available: www.canada.ca/en/public-health/services/diseases/2019 -novel-coronavirus-infection.html? \&utm_campaign=gc-hc-sc-coronaviruspublic edu2021-2021-0001-9762248618\&utm_medium=search\&utm_source=google -ads-99837326356\&utm_content=text-en-428935858540\&utm_term=covid (accessed 2021 Apr. 1)

8. Shoukat A, Wells CR, Langley JM, et al. Projecting demand for critical care beds during COVID-19 outbreaks in Canada. CMA7 2020;192:E489-96.

9. Tuite AR, Fisman DN, Greer AL. Mathematical modelling of COVID-19 transmission and mitigation strategies in the population of Ontario, Canada. CMA7 2020;192:E497-505.

10. McMorrow T. Interpreting eligibility under the medical assistance in dying law: the experiences of physicians and nurse practitioners. McGill 7 Law Health 2020 Oct. 6.

11. Dicicco-Bloom B, Crabtree BF. The qualitative research interview. Med Educ 2006; 40:314-21.

12. Lipscomb M. Abductive reasoning and qualitative research. Nurs Philos 2012;13:244-56.

13. Wallace CL, Wladkowski S, Gibson A, et al. Grief during the COVID-19 pandemic: considerations for palliative care providers. 7 Pain Symptom Manage 2020;60:e70-e76.

14. Etkind SN, Bone AE, Lovell N, et al. The role and response of palliative care and hospice services in epidemics and pandemics: a rapid review to inform practice during the COVID-19 pandemic. 7 Pain Symptom Manage 2020;60:e31-e40
15. de Haes H, Teunissen S. Communication in palliative care: a review of recent literature. Curr Opin Oncol 2005;17:345-50.

16. Find an independent witness for medical assistance in dying. Toronto: Dying with Dignity Canada. Available: www.dyingwithdignity.ca/witness_program (accessed 2020 Apr. 17).

17. Grant K. Medical assistance in dying services being cancelled in Ottawa, Hamilton areas. The Globe and Mail [Toronto]; 2020 Mar. 27. Available: www.theglobeandmail.com/canada/article-medical-assistance-in-dying-services -being-cancelled-in-ottawa/ (accessed 2020 June 5).

18. Dion S, Wiebe E, Kelly M. Quality of care with telemedicine for medical assistance in dying eligibility assessments: a qualitative study. CMAJ Open 2019;7:E721-9

19. Changes to the MAiD process in response to the COVID-19 pandemic. Vancouver: College of Pharmacists of British Columbia; 2020. Available: www.bcpharmacists.org/medical-assistance-dying (accessed 2020 June 6).

Affiliations: Department of Family Medicine (Wiebe), University of British Columbia, Vancouver, BC; University of London (Kelly), London, UK; University of Ontario Institute of Technology (McMorrow), Oshawa, Ont.; Université de Sherbrooke (Tremblay-Huet), Sherbrooke, Que.; University of British Columbia (Sum, Hennawy), Vancouver, BC

Contributors: Ellen Wiebe conceived and designed the study. Michaela Kelly, Sabrina Tremblay-Huet and Brian Sum acquired the data, which all authors analyzed and interpreted. Ellen Wiebe drafted the first version of the manuscript. All authors critically revised the manuscript, gave approval of the final version and agreed to be accountable for all aspects of the work.

Content licence: This is an Open Access article distributed in accordance with the terms of the Creative Commons Attribution (CC BY-NC-ND 4.0) licence, which permits use, distribution and reproduction in any medium, provided that the original publication is properly cited, the use is noncommercial (i.e., research or educational use), and no modifications or adaptations are made. See: https://creativecommons.org/licenses/by-nc-nd/4.0/

Data sharing: The data collected in this study are not suitable for sharing beyond the information provided in this article. Further information can be obtained from the corresponding author.

Supplemental information: For reviewer comments and the original submission of this manuscript, please see www.cmajopen.ca/content/9/2/ E400/suppl/DC1. 